\title{
Cooperative Diversity in Carrier Frequency Offset
}

\author{
A. Özgür Y1lmaz
}

\begin{abstract}
Cooperative diversity can enjoy the same benefits of MIMO systems when there is no possibility of carrying multiple antennas on a communication terminal. The main distinction between MIMO and cooperative diversity systems is the inherent asynchronism in the latter. We propose a model to study the effects of carrier frequency offset along with symbol asynchronism. Based on the proposed model, a Viterbi equalizer is utilized as a solution for demodulation. Through simulations, it is observed that such a solution suffers a graceful loss with increasing carrier frequency offset.
\end{abstract}

\section{INTRODUCTION}

Diversity is a powerful technique to mitigate the adverse effects of fading in wireless communications. Space diversity made possible by multi-input multi-output (MIMO) systems [1], [2] have been quite popular in the last decade. Although MIMO systems provide substantial increase in capacity, it may not always be possible to physically carry multiple antennas at transmitters and receivers. This problem can be solved by distributed antenna systems formed by antennas belonging to different terminals [3]. After a communication with relay terminals, a virtual antenna array consisting of the antennas of the relays can be created and utilized in analogy with a MIMO system.

Many of the techniques developed for MIMO systems can be easily extended to be used in cooperative diversity [4], [5]. However, most of the research work assume a perfectly synchronous communication scenario which somehow masks the difference of cooperative diversity schemes as to MIMO. In MIMO systems, all the antennas at the transmitter are fed with the same clock and hence there is perfect synchronization. The same holds for the receiver side. In cooperative diversity we cannot talk of the same level of synchronization since the antennas are on different terminals. Hence, cooperating antennas are running with different clocks and this constitutes the main distinction of cooperative diversity as compared to MIMO systems.

There could be various sorts of synchronization problems in a communication system. We neglect the frame synchronization problem but focus on symbol and local oscillator synchronization in this article. Cooperative diversity with no symbol synchronization can be studied in the form of an intersymbol interference (ISI) channel as in [6] where an MMSE based solution was proposed. In [7] a family of spacetime trellis codes were utilized to resolve the same problem. We will utilize the ISI channel model in order to take the symbol asynchrony into account. We will further induce a

The author is with the Department of Electrical and Electronics Engineering, Middle East Technical University, Ankara, Turkey (e-mail: aoyilmaz@metu.edu.tr). carrier frequency offset on the signals coming from the relays which can occur due to missynchronized local oscillators, mobility of the terminals etc.

There are studies that evaluate the capacity of cooperative diversity within various scenarios [3], [8], [9]. Current literature seems to lack of performance evaluation of practical cooperative schemes. We focus, in this study, on a practical scenario where relays perform the simple decode-and-forward operation without any manipulation on data where perfect synchronization is not assumed. The outline is as follows. We explain the system model in Section II. The proposed equalizer is described in Section III along with performance evaluation. The paper is concluded with Section IV.

\section{CoOperation Model}

We consider a cooperative diversity scheme in which a source transmits its signal uncoded in packets to relays first with appending cyclic redundancy check (CRC) bits. The relays demodulate the signal sent by the source and transmit it to a destination without any modification as in repetition coding if CRC check is successful. We consider such a plain uncoded scenario along with flat fading channels to closely observe the effects of carrier frequency offset. Other scenarios can be studied within a similar framework. Root raised cosine (RRC) pulse shaping and binary phase shift keying (BPSK) modulation is used throughout the study.

At the destination, the signal received from a relay $i$ is

$$
x_{i}^{\prime \prime}(t)=\Re\left\{e^{j 2 \pi\left(f_{c}+f_{i}\right) t} \alpha_{i} \sum_{k=0}^{N-1} u_{k} g\left(t-k T_{s}-\tau_{i}\right)\right\},
$$

where $f_{c}$ is the desired carrier frequency, $f_{i}$ is the carrier frequency offset for relay $i, \alpha_{i}$ is the channel coefficient from relay $i$ to destination, $N$ is the number of symbols in a packet, $u_{k}$ 's are transmitted symbols, $g(t)$ is the RRC pulse, $T_{s}$ is the symbol duration, and $\tau_{i}$ is the time offset. The channel coefficient $\alpha_{i}$ is a circularly symmetric complex Gaussian zero mean random variable with variance 1 . The RRC pulse is truncated to $\left[-6 T_{s}, 6 T_{s}\right]$. This signal is downconverted by $f_{c}$ and after I/Q demodulation the equivalent lowpass signal is

$$
x_{i}^{\prime}(t)=e^{j 2 \pi f_{i} t} \alpha_{i} \sum_{k=0}^{N-1} u_{k} g\left(t-k T_{s}-\tau_{i}\right) .
$$

A standard matched filtering operation is performed with $g(-t)$ and hence

$$
x_{i}(t)=\sum_{k=0}^{N-1} u_{k} \alpha_{i}\left(e^{j 2 \pi f_{i} t} g\left(t-k T_{s}-\tau_{i}\right)\right) * g(-t)
$$

is obtained where $*$ stands for the convolution operation. Had not been any carrier frequency offset and any problem with 
respect to sampling time offset, a sampling period of $T_{s}$ would have ensured an ISI-free discrete baseband equivalent signal. Since there are multiple relays whose signals interfere at the destination, it is possible neither to resolve carrier frequency nor sampling time offset problems.

In general, the term $\left(e^{j 2 \pi f_{i} t} g(t-\tau)\right) * g(-t)$ cannot be simplified. However, when $f_{i} T_{s}$ is not so large, it can be checked numerically that a good approximation is

$$
\left(e^{j 2 \pi f_{i} t} g(t-\tau)\right) * g(-t) \approx e^{j 2 \pi f_{i} t}(g(t-\tau) * g(-t)) .
$$

We will develop a model to be utilized in the remainder of the paper using this approximation while signals in simulations will be generated based on the actual signal in (3). Upon the approximation above, the signal received from relay $i$ at destination becomes

$$
x_{i}(t)=\sum_{k=0}^{N-1} u_{k} \alpha_{i} e^{j 2 \pi f_{i} t} r\left(t-k T_{s}-\tau_{i}\right),
$$

where $r(t-\tau)=g(t-\tau) * g(-t)$.

After the sampling operation, a discrete-time version of the signal is obtained and a received vector is formed by $x_{i, n}=$ $x_{i}\left(n T_{s}\right)$. ISI results from the $r\left(t-\tau_{i}\right)$ after sampling due to sampling time offset. We will make use of an $L$-tap vector

$\left[h_{0}^{i} h_{1}^{i} \ldots h_{L-1}^{i}\right]=\alpha_{i}\left[r\left(-\tau_{i}\right) r\left(T_{s}-\tau_{i}\right) \ldots r\left((L-1) T_{s}-\tau_{i}\right)\right]$

to represent this ISI with the channel gain. When all these are combined, the following expression is found with the vector

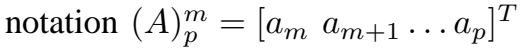

$$
\left(X_{i}\right)_{p}^{m}=\delta_{i}^{m} D_{i} H_{i}(U)_{p}^{m-L+1}
$$

where $\delta_{i}=e^{j 2 \pi T_{s}}, D_{i}$ is a diagonal matrix with elements $1, \delta_{i}, \delta_{i}^{2}, \ldots, \delta_{i}^{p-m}$, and $H_{i}$ is the $(p-m+1) \times(p-m+L)$ convolution matrix corresponding to $\left[h_{L-1}^{i} h_{L-2}^{i} \ldots h_{0}^{i}\right]$. Perfect channel state information is assumed to be available at the destination. This information includes both the frequency offset and the ISI due to sampling time offset. Hence, the matrices $D_{i}$ and $H_{i}$ are perfectly known at the destination. One possible way to estimate these matrices is to send a training sequence repetitively a few times and estimate the Doppler frequency just as it is done in Doppler radars [10]. This sort of a channel identification is beyond the scope of this paper and will be addressed in future studies.

For the remainder of this paper, two relays will be sending signals to the destination. When this occurs, we have the following received signal at the destination

$$
(Y)_{p}^{m}=\left(\delta_{0}^{m} D_{0} H_{0}+\delta_{1}^{m} D_{1} H_{1}\right)(U)_{p}^{m-L+1}+(W)_{p}^{m},
$$

where $Y$ and $W$ vectors stand for the received signal and circularly symmetric complex Gaussian white noise with zero mean and variance $N_{0}$, respectively. If an MMSE solution is to be used to estimate the transmitted symbols, one has to use the autocorrelation of $(Y)_{p}^{m}$ which can be evaluated as

$$
\begin{aligned}
E\left[(Y)_{p}^{m}\left((Y)_{p}^{m}\right)^{H}\right] & =E_{s} \cdot\left(D_{0} H_{0} H_{0}^{H} D_{0}^{H}+D_{1} H_{1} H_{1}^{H} D_{1}^{H}\right. \\
& \left.+2 \Re\left\{\left(\delta_{0} / \delta_{1}\right)^{m} D_{0} H_{0} H_{1}^{H} D_{1}^{H}\right\}\right)+N_{0} I,
\end{aligned}
$$

where $(\cdot)^{H}$ denotes the conjugate transpose and $I$ is the identity matrix. It is obvious from (9) that the autocorrelation and hence the MMSE filter change in time due to the $\left(\delta_{0} / \delta_{1}\right)^{m}$ term. Hence, the approach taken in [6] is not helpful when there is carrier frequency offset in a cooperative diversity scheme. A Viterbi algorithm [11] based solution is to be proposed and its performance evaluated in the next section.

\section{Viterbi Equalizer in CARRIER FrequenCy OFFSET}

Through (8), the received signal at time epoch $m$ can be expressed as

$$
y_{m}=\sum_{k=0}^{L-1}\left(\delta_{0}^{m} h_{k}^{0}+\delta_{1}^{m} h_{k}^{1}\right) u_{m-k}+w_{k} .
$$

Keeping the symbols prior to the current symbol in a state, a state trellis can be constructed just as in the case of regular Viterbi based ISI equalizers. The only modification to be made is on the channel coefficients. As opposed to the regular ISI, we also have rotating phasor terms $\delta_{0}$ and $\delta_{1}$. These terms are raised to the $m^{t h}$ power, multiplied by the channel coefficients for the respective relays, and overall channel coefficients at epoch $m$ are calculated as a sum of these two channel coefficient vectors. These channel coefficients are then used in branch metric calculation. This modification adds a negligible computational complexity to a Viterbi equalizer.

Two values of the RRC rolloff factor $\beta$ will be made use in simulations. The first value is 0.5 which corresponds to a more spread spectrum in comparison to the second value of 0.2 which forms a quite confined spectrum. Two relays cooperate in all the simulations with perfect channel information at destination. Without loss of generality, it is assumed that the relay with the smaller delay $\tau_{i}$ has no carrier frequency offset. To incorporate the effect of sampling offset, it is assumed that sampling starts at $t=0$ secs while the relays have random delays $\tau_{0}$ and $\tau_{1}$. Moreover, it is assumed that $\tau_{0}$ is uniformly distributed within $\left[0,0.5 T_{s}\right]$ secs so that sampling starts around the arrival of the first relay's signal as that would occur in real life. We furthermore consider such a network scenario that the delays between two relays is uniformly distributed between $\left[0, T_{s}\right]$ secs so that the time offset between two relays do not exceed one symbol period. A tap number of $L=3$ is used under the scenario investigated here. All these numbers are arbitrary and can be changed as desired. However, one should take into account the fact that a higher number of taps and thus a more complex equalizer have to be utilized if relays cause a more spread channel. In that case, data rate can be traded off against computational complexity.

In Fig. 1 the bit error rate (BER) of BPSK modulation equalized by the algorithm explained above is depicted. Different lines correspond to different values of carrier frequency offsets normalized with the symbol duration. For example, a carrier frequency offset of $500 \mathrm{kHz}$ correspond to a normalized frequency offset of 0.05 if the symbol duration equals 100 nsecs. Data symbols are transmitted in packets of length 100. (9) At least 1000 packet errors are collected for each simulation point. As seen in the figure, the algorithm works quite well up until a normalized carrier frequency of 0.06 . SNR losses range around 0 to $0.3 \mathrm{~dB}$ for carrier offsets up to 0.06 when compared 
to the case of no carrier offset. However, at larger frequency offsets SNR losses are around $1-2 \mathrm{~dB}$ depending on the operation point in the SNR range of interest. This undesirable loss is due to the inaccuracy of the approximation in (4) at high frequency offset values. Also drawn in the figure for comparison are the error rate curves for $\tau_{0}=\tau_{1}=0$ and $\tau_{0}=$ $0, \tau_{1}=T_{s}$ cases, both with no carrier frequency offset. The former one corresponds to perfectly synchronized relays and offers a diversity order of one along with a channel coefficient with twice the power. The diversity order is not two since a repetition coding is implemented in this study rather than an Alamouti-like code. The latter case has diversity two as there are two perfectly distinguishable taps in the channel response. Error rates are analytically calculated based on the BPSK error performance in fading channels by Thm 4.1 in [2]. The performance of the system studied here exhibit performance in between these two cases since $\tau_{1}-\tau_{0}$ is uniformly distributed in $\left[0, T_{s}\right]$ and thus has diversity in average between one and two.

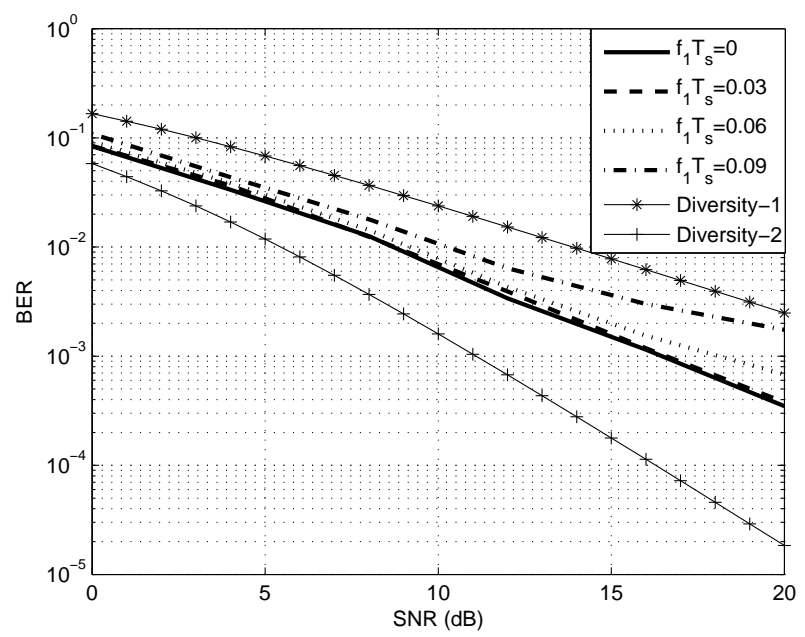

Fig. 1. Bir error rate performance of the proposed equalizer at various carrier frequency offset values

A more confined spectrum should exacerbate the problem occurring due to frequency offset. When there is frequency offset, the signal spectrum shifts. The matched filter spectrum does not perfectly align with the shifted signal spectrum. Hence, there has to be a larger SNR loss in smaller values of rolloff factor. In Fig. 2 BER is depicted for the previous scenario with $\beta=0.2$. In this case, SNR losses are more visible with increasing frequency offsets as expected.

\section{CONCLUSION}

Asynchronism is inherent in cooperative diversity systems. We propose a model to study the effects of imperfect carrier frequency and symbol synchronization. An algorithm based on Viterbi algorithm is developed and its performance is evaluated by Monte Carlo simulations. It is found out that the proposed algorithm effectively mitigates the carrier frequency offset problem unless the offset is large. Moreover, carrier frequency offset degrades the performance to a larger extent when the

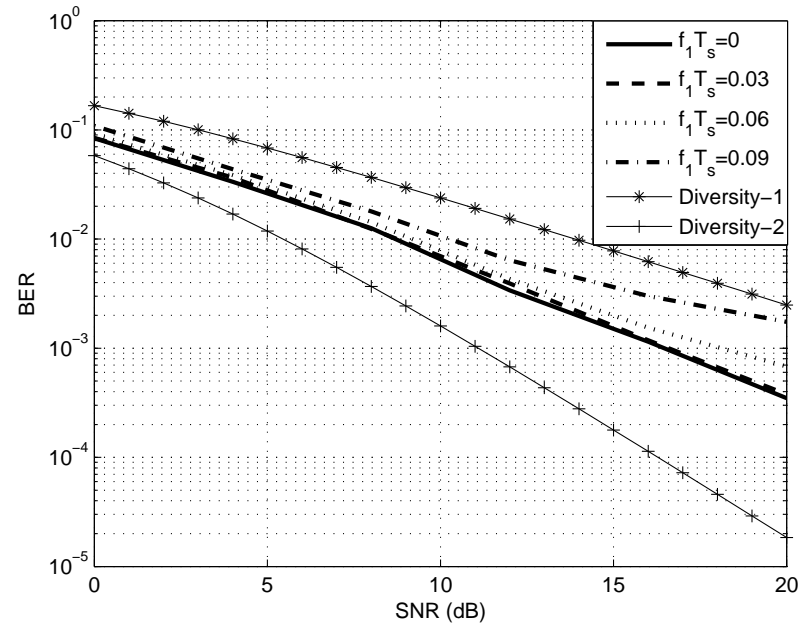

Fig. 2. Bit error rate performance of the proposed equalizer at various carrier frequency offset values

transmitted signal has a more compact spectrum. We currently address the channel identification problem in the system studied here along with research on analytical performance evaluation, use of differential modulation in such a setting, other approximations for the frequency-shifted signals, and space-time coding.

\section{REFERENCES}

[1] S.M. Alamouti, "A simple transmit diversity technique for wireless communications," IEEE J. Sel. Areas Comm., vol. 16, no. 8, pp. 14511458, October 1998.

[2] E.G. Larsson and P. Stoica, Space-Time Block Coding for Wireless Communications, Cambridge University Press, 2003.

[3] J.N. Laneman, D.N.C. Tse, and G.W. Wornell, "Cooperative diversity in wireless networks: Efficient protocols and outage behavior," IEEE Trans. on Information Theory, vol. 50, no. 12, pp. 3062 - 3080, December 2004.

[4] I. Hammerstroem, M. Kuhn, B. Rankov, and A. Wittneben, "Spacetime processing for cooperative relay networks," Vehicular Technology Conference, vol. 1, pp. 404-408, October 2003.

[5] A. Scaglione and Y. Hong, "Opportunistic large arrays: Cooperative transmission in wireless multihop ad hoc networks to reach far distances," IEEE Transactions on Signal Processing, vol. 51, no. 8, pp. 2082-2092, August 2003.

[6] S. Wei, D. Goeckel, and M. Valenti, "Asynchronous cooperative diversity," Proc. Conf. Inform. Sci. and Sys., March 2004.

[7] Y. Li and X. Xia, "A family of distributed space-time trellis codes with ascynchronous cooperative diversity," Information Processing in Sensor Networks, pp. 340-347, April 2005.

[8] R.U. Nabar, H. Bolcskei, and F.W. Kneubuhler, "Fading relay channels: performance limits and space-time signal design," IEEE J. Sel. Areas Comm., vol. 22, no. 6, pp. 1099-1109, August 2004.

[9] A. Host-Madsen and J. Zhang, "Capacity bounds and power allocation in wireless relay channel," IEEE Trans. on Information Theory, vol. 52, no. 4, pp. 1522-1544, April 2006.

[10] M.I. Skolnik, Introduction to Radar Systems, McGraw-Hill, 2001.

[11] J.G. Proakis, Digital Communications, McGraw-Hill, 1995. 